\title{
Syncope Beginning in People Over 50 Years old-Experience in 52 Cases
}

\author{
Jiménez-Cohl Pedro¹, Aspeé Maximiliano², Vega M. Teresa², Canto Yianinna², Peña José Tomás², \\ Bocaz Sebastián ${ }^{2}$, Tapia Francisco ${ }^{2}$
}

${ }^{1}$ Physician Neurology Service, Militar Hospital of Santiago (Chile), University of Valparaíso, Campus San Felipe, San Felipe, Chile ${ }^{2}$ University of Valparaíso, Campus San Felipe, San Felipe, Chile

Email: pejimco@yahoo.com

How to cite this paper: Pedro, J.-C., Maximiliano, A., Teresa, V.M., Yianinna, C., Tomás, P.J., Sebastián, B. and Francisco, T. (2020) Syncope Beginning in People Over 50 Years Old-Experience in 52 Cases. International Journal of Clinical Medicine, 11, 439-453.

https://doi.org/10.4236/ijcm.2020.117037

Received: May 23, 2020

Accepted: July 12, 2020

Published: July 15, 2020

Copyright $\odot 2020$ by author(s) and Scientific Research Publishing Inc. This work is licensed under the Creative Commons Attribution International License (CC BY 4.0).

http://creativecommons.org/licenses/by/4.0/

\begin{abstract}
It is not common to start suffering from syncopes after age 50. They are mainly male patients who present causes other than vasovagal syncope, which predominates at an early age. Orthostatic hypotension is the predominant causal factor, which is attributed in many cases to advanced age, metabolic, cardiovascular or neurological diseases, to failure of baroreflexes, all of the above may be associated with the use of hypotensive drugs alone or in combination with psychotropic drugs. Furthermore, causes such as carotid sinus syncope, postprandial syncope and situational syncope become more frequent. Therefore, as people age, they present a favorable pathological terrain for the production of syncope. The older you are, the more likely you are to start with syncope. Finding the definitive diagnosis for their syncopes can be difficult, given the multiplicity of interacting factors. Their study is more exhaustive and requires a good anamnesis, knowing the drugs used by the patient, concomitant diseases and careful surveillance to get closer to the diagnosis.
\end{abstract}

\section{Keywords}

Vasovagal Syncope, Fainting, Dysautonomia, Older Adults

\section{Introduction}

Syncope and/or lipothymia are common at all ages. However, going over 50 and just starting to present them is unusual. The first peak is during adolescence at age 15 [1]. Its frequency is usually twice as high in women as in men [2] [3]. Neurally mediated reflex syncope (vasovagal syncope (VVS)) predominates as the first cause of these [2] [3] [4]. 
There is another peak, somewhat smaller after 65 years [4] [5] [6]. This group is special, since other causes for syncope appear and it is more complex to find a single cause, because there may be more than one, in the same person. $40 \%$ $60 \%$ of cases, could be multicausal [7] [8] [9].

We show our results, in terms of sex, age, associated factors, probable causes, and follow-up, in 52 patients who started their syncopes at age 50 or later. They were sent to us after a cardiac cause was ruled out and there were doubts about the cause of the condition. They were studied with head up tilt (HUT) from 2012 to 2018 and followed during their evolution, between 15 months and 7 years (Mean age: 4.5 years).

\section{Material and Methods}

\section{Pre-Tilt test study and inclusion criteria}

This study included retrospective data analysis of 212 patients studied with HUT (60\% female), conducted between 2012 and 2018. The mean age of these patients was 31.8 years (range: 6 - 89 years).

Only 52 subjects (25\%) who consulted for syncope or presyncope, beginning at 50 years or older were included. None had a history of prior syncope.

To rule out a cardiac or other causes, subjects were screened with a medical history, physical examination, an evaluation by a cardiologist and examinations: electrocardiogram (12 lead), echocardiogram, heart rate holter, and sometimes an electrophysiological study. If this review is negative or doubtful, the patient is sent for our neurological evaluation and HUT.

If a cardiac cause was proven the patient was not included. The same if the patient was epileptic or suffered from pseudosyncopes. 80 volunteers of the same age and sex served as controls.

Subjects were recruited from patients of the Militar Hospital from Santiago.

\section{Tilt Test Exam Conditions}

Fasting patient, between 8 and 12 hours. Quiet room, with dim light at a temperature between $20^{\circ} \mathrm{C}-22^{\circ} \mathrm{C}$. Supervised by a neurologist, a cardiologist and a medical technologist. Cardiology personnel installs continuous EKG monitoring.

A hemoglucotest is performed prior to the exam.

Electrocardiographic monitoring and continuous measurement of blood pressure, heart rate and surveillance of doctors and nurses are performed.

Drugs, venous lines, a defibrillator, and equipment for cardiopulmonary resuscitation are available.

\section{Tilt Test Protocol}

A record of heart rate (HR) and blood pressure (BP) and of symptoms reported by the patient is kept every 5 minutes. The reason for stopping the examination or any important incident is noted and recorded at any time. The sublingual ni- 
troglycerin protocol is based on Del Rosso [10].

Carotid massage is performed on all patients over 60 years of age. Previous discard of murmur or carotid stenosis or stroke in the last 6 months. Five minutes on each side [11].

Tilt Test ends if a "positive HUT" is obtained: This is syncope (loss of consciousness) or presyncope (dizziness, nausea, paleness, etc., announcing that syncope is imminent). Associated with low blood pressure (systolic BP $<70$ $\mathrm{mmHg}$ ) or low blood pressure plus bradycardia, or if intolerable patient discomfort occurs.

If there are no symptoms, it is terminated due to the end of the protocol.

In addition, sympathetic and parasympathetic function tests (Valsalva maneuver and deep breathing test) are performed in order to support or rule out failure in the baroreflexes.

The equipment consists of: Digital monitor (Ohmeda 2300 Finapres BP Monitor USA). Digital cuff placed on the index or middle finger to measure BP and HR continuously.

Electric tilting table (Magnetic Manumed USA) and electrocardiogram monitor (Quinton Q4500 USA). The patient is fastened to the table with two velcro straps (knees and chest).

For the statistical comparison, Anova and logistic regression are used, and depending on the sample size, a non-parametric test is used.

\section{Ethical Clearance}

Our study was analyzed and approved by the institutional ethics committee of the Hospital Militar, and was carried out in accordance with the ethical standards of the Helsinki Declaration 1964. Patients and controls signed an informed consent before inclusion.

\section{Results}

In 52 syncope started from the age of 50 or later, 34 men (64\%). Mean age 67.5 years (range: 50 - 89 years). Of these, 54\% were 65 or older (range: 65 - 89 years/43\% female). Table 1 shows the patients according to age and sex.

Table 2 shows the number of patients and the age of onset of their syncopes by decade.

The predominance of males is clear in these adults who have just started their syncope, even worse after 65 years ( $57 \%$ at that age). The opposite occurs in the youth population [1] [2] [3] [4] [12].

The reasons for consultation in our patients were: syncope, lipothymia, orthostatic dizziness and different combinations between them. All these account for $88 \%$ of cases (n: 46), which is comparable to the youth population [1] [2] [3] [4] [12] [13].

Table 3 shows the reason for consultation of the patients and the positivity of the HUT. 
Table 1. Sex and age of onset of syncopes.

\begin{tabular}{cccc}
\hline \multicolumn{4}{c}{ Sex and age at the onset of symptoms } \\
\hline & Female & Male & Total \\
\hline $\mathrm{N}^{\circ}$ of cases & 18 cases & 34 cases & 52 cases \\
Mean Age & 69.9 years & 66.1 years & 67.5 years \\
\hline
\end{tabular}

Table 2. Number of patients and age of onset of their syncopes by decade.

\begin{tabular}{ccc}
\hline \multicolumn{3}{c}{ Number of cases and age of onset of syncope per decade } \\
\hline Age (years) & $\mathrm{N}^{\circ}$ of cases & $\%$ \\
\hline $50-59$ & 18 patients & $34 \%$ \\
$60-69$ & 10 patients & $19 \%$ \\
$70-79$ & 15 patients & $29 \%$ \\
$80-89$ & 9 patients & $17 \%$ \\
TOTAL & 52 cases & $100 \%$ \\
\hline
\end{tabular}

Table 3. Reason for consultation and Tilt Test result in 52 patients.

\begin{tabular}{cccc}
\hline Reason for consultation & $\mathrm{N}^{\circ}$ of cases & HUT positive \% & HUT Negative \% \\
\hline Syncopes & 26 & $20(77 \%)$ & $6(23 \%)$ \\
Syncopes and lipothymia & 7 & $6(86 \%)$ & $1(14 \%)$ \\
Lipothymia & 6 & $6(100 \%)$ & Zero \\
Postprandial syncope & 5 & $4(80 \%)$ & $1(20 \%)$ \\
Postural dizziness & 4 & $3(75 \%)$ & $1(25 \%)$ \\
Dizziness and syncopes & 1 & $1(100 \%)$ & Zero \\
Convulsive syncope & 1 & $1(100 \%)$ & Zero \\
Laziness and drowsiness & 1 & Zero & $1(100 \%)$ \\
Parkinson and dizziness & 1 & Zero & $1(100 \%)$ \\
Total & $\mathrm{N}: 52(100 \%)$ & N: $41(79 \%)$ & N: $11(21 \%)$ \\
\hline
\end{tabular}

\section{Findings in the Tilt Test}

The overall positivity of the HUT was $78 \%$, (n: 41 ), which is common in patients selected by trained doctors and sent to HUT [14] [15] [16].

The most important final events found in the positive HUT were: symptomatic orthostatic hypotension $(\mathrm{OH})$ (n: 24 cases $/ 46 \%$ ), with final collapse of the patient. Followed by mixed vagal syncope mainly vasodilator n: $11(21 \%)$ and cardioinhibitory vagal syncope with $8 \%$ (n: 4). This is shown in Table 4. 
Negative Tilt Test: Eleven patients (21\%), who consulted for syncope, lipothymia, or orthostatic dizziness had a negative HUT. They were followed up, trying to find a possible cause of their symptoms.

The possible explanation of symptoms in patients with negative HUT can be seen in Table 5.

\section{Orthostatic Hypotension $(\mathrm{OH})$}

It is frequently found in our patients n: 24 (46\%).

Hypotension can occur early, within the first 3 minutes ( 4 cases) or more frequently after 3 minutes (late OH), in 20 patients [17] [18] [19].

To consider it as the cause of the condition, hypotension must produce orthostatic symptoms during the HUT [19].

Table 6 shows the number of patients and the probable cause of $\mathrm{OH}$, postulated after an average follow-up of 4.5 years. The $\mathrm{n}$ is greater than 24 , because

Table 4. Tilt Test results 52 patients.

\begin{tabular}{ccc}
\hline Results & $\mathrm{N}^{\circ}$ & $\%$ \\
\hline Orthostatic hypotension & 24 & $46 \%$ \\
Vagal syncope > vasodilator & 11 & $21 \%$ \\
Negative Tilt Test & 11 & $21 \%$ \\
Vagal syncope cardio inhibitory & 4 & $8 \%$ \\
Carotid sinus syncope & 2 & $4 \%$ \\
TOTAL & 52 & $100 \%$ \\
\hline
\end{tabular}

Table 5. Possible etiological explanation of consultation symptoms in patients with a negative Tilt Test (HUT).

\begin{tabular}{|c|c|c|c|c|}
\hline Sex & Age & Reason of Consultation & Explanation & $\mathrm{N}$ \\
\hline Male & 63 & $\begin{array}{c}\text { Parkinson's disease } \\
\text { screening }\end{array}$ & $\begin{array}{c}\text { Parkinson's disease with } \\
\text { no dysautonomia }\end{array}$ & 1 \\
\hline Female & 76 & Syncope & Cardiac arrythmia & 1 \\
\hline Male & 57 & Cough and syncope & Cough Syncope & 1 \\
\hline Male & 56 & Daytime sleepiness & Sleep Apnea & 1 \\
\hline Female and Male & 76 and 52 & Postural dizziness & $\begin{array}{c}\text { Stroke }+ \text { High blood } \\
\text { pressure (uncontrolled) }\end{array}$ & 2 \\
\hline Female & 66 & Syncope & Drug effect & 1 \\
\hline Male & 65 & Syncope after feeding & Postprandial syncope & 1 \\
\hline Male (both) & 62 and 71 & Syncope and dizziness & Carotid sinus syncope & 2 \\
\hline Male & 59 & Orthostatic dizziness & Not explained & 1 \\
\hline
\end{tabular}


Table 6. Factors associated with the existence of orthostatic hypotension.

\begin{tabular}{cccc}
\hline Factors associated with $\mathrm{OH}$ & $\mathrm{N}$ & $\%$ & Mean age \\
\hline Cardiovascular diseases & 13 & 25 & 78 \\
Hypotensive drugs & 13 & 25 & 72 \\
Baroreflexes failure* & 8 & 15 & 75 \\
Autonomic NS neuropathy & 3 & 6.0 & 72 \\
Joint hypermobility $\geq 7$ & 2 & 4.0 & 65 \\
Postprandial syncope & 1 & 2.0 & 73 \\
Diabetic neuropathy & 3 & 2.0 & 63 \\
No apparent explanation & 5 & 10 & 68 \\
Total combinations & 48 & $89 \%$ & 70.5 years \\
\hline
\end{tabular}

${ }^{\star}$ Includes 3 patients with Parkinson's disease.

several factors were combined in most of the patients, without being able to assure which is the influence that each one of them weighs. 48 possible combinations were found, which would explain $89 \%$ of the causes of $\mathrm{OH}$.

In 5 patients with clear $\mathrm{OH}$, we were unable to find any associated concomitant factors.

\section{Drug Use and Orthostatic Hypotension}

19 patients (36\%) took medications potentially related to the production of $\mathrm{OH}$ and syncope or lipothymia: Antihypertensive, antidepressant or antipsychotic drugs [7] [20].

Antihypertensive drugs found were losartan $\mathrm{n}=6$ (11\%), enalapril 5 (10\%) and atenolol $4(8 \%)$. Combined with diuretics, or with psychotropic drugs $n=4$ $(8 \%)$.

Antipsychotics or antidepressants used were: amitriptyline, trazodone, haloperidol and quetiapine. All were combined with antihypertensive drugs (n: 4). Five patients were using sertraline, but their syncopes had already started before starting the drug.

Eight of these patients (42\%) benefited from the withdrawal or decrease of antihypertensive drugs.

\section{Cardiovascular Comorbidities}

In 31 patients we found comorbidities in the cardiovascular area (60\%), and the use of drugs related to that area.

High blood pressure (HBP) 20 cases, diabetes mellitus (DM) 10 cases, stroke 5 cases, heart failure 2 cases, pacemaker 1 case, arrhythmia one case, dyslipidemia 3 cases, and different combinations between them (n: 23). 


\section{The Deterioration or Loss of the Baroreflexes}

In 10 cases ( $24 \%$ of positive HUTs), we found damage to the baroreflexes, corroborated as orthostatic hypotension, without accompanying tachycardia [21] plus abnormalities in blood pressure response in the Valsalva Maneuver and in deep breathing test [22] [23].

\section{Situational Syncope}

We found two patients with situational syncope. Both males. One of them when coughing presented syncope with severe drop in BP and HR, consistent with his symptoms ("cough syncope"). However, his orthostatic HUT was negative.

The other case presented episodes of very prolonged laughter ("gelastic syncope"), with choking and syncope. His orthostatic HUT was positive (vasodilator).

\section{Postprandial Syncope}

We found postprandial syncope (PPS) in 5 patients (9.6\%). Mean age: 71.2 years. Four males. Syncope only appeared after feeding. Four with positive HUT (3 with vasodilator VVS and one with $\mathrm{OH}$ ). They all suffered from HBP. Three were type 2 diabetics and two had hypercholesterolemia. In patients under 60 years of age, we have only found one case of postprandial syncope.

\section{Carotid Sinus Syncope}

Carotid sinus compression was practiced in all patients 60 years of age or older, according to what international protocols dictate [11].

Compression was positive in 2 males (4\%). Mean age 66 years. Both had a negative orthostatic HUT for vasovagal dysautonomia. They consulted for presenting dizziness or syncope when changes in posture of the head. This endorses the usefulness of using such compression in the study of syncope in older adults. [11].

\section{Parkinson's Disease}

Four patients ( 3 males) Mean age: 78 years. Three with Parkinson's over 7 years of evolution, in treatment associated with hypotensive drugs. Sent by syncopes and lipothymia.

All three with failure in the baroreflexes and positive HUT for $\mathrm{OH}$.

In one with recent Parkinson's (3 years). HUT was performed to rule out dysautonomia. He did not suffer from syncope or lipothymia. His HUT was negative.

\section{A Special Case: Patients over 65}

They are the bulk of our cases, $\mathrm{n}=28$ (54\%). 16 males $(57 \%)$.

These patients suffer more frequently of: syncope (78\%), orthostatic hypoten- 
sion (85\%), cardiovascular comorbidities (68\%), postprandial syncope $(9.6 \%)$, failure of baroreflexes (28\%), use of hypotensive drugs (53\%), concomitant diseases such as Parkinson's (14\%), carotid sinus syncope (3.5\%) and many associations between these factors.

\section{Follow Up}

This covers between 15 months and 7 years (X: 4.5 years). It allowed us to find causes that are unusual at younger ages: Use of hypotensive drugs (n: 8), one of them, added a diabetic autonomic neuropathy. Postprandial syncope (n: 4), post-stroke dizziness (n: 1). Arrhythmia (n: 1). Carotid sinus syndrome (n: 2). Sleep apnea with hypersomnia (n: 1) and degenerative neurological diseases such as Parkinson's disease with dysautonomia (n: 3 ). The total for this type of unusual cause is 20 cases $(38 \%)$.

\section{Unexplained Cases}

Despite follow-up and complementary examinations, there were 6 patients (11\%), in whom we did not find associated factors that made them prone to syncope.

They all had a positive HUT. Three with $\mathrm{OH}$ and three with vasovagal syncope.

In three of them, syncope occurred only once.

It is known that $10 \%$ of syncopes remain unexplained in any statistic [24] [25] [26].

\section{Discussion}

Approximately $50 \%$ of people present their first fainting, between 15 and 30 years of age [1], at that age, its frequency is usually double in women and neurally mediated syncope (VVS) predominates as the first cause [2] [3].

But up to $10 \%$ to $15 \%$ of the population would have it after the age of 65 [3].

In our patients over 50 years old, the relationship of sex is reversed, with the frequency being much higher in men (63\% vs. 38\%) than in women [27] [28].

The most important final event in HUT was orthostatic hypotension (46\%). Vasovagal syncope is relegated to second place with $29 \%$. This supports the observation that there are different causes for syncope in these patients than in young people [2] [4] [5] [6].

Certain factors alone or associated could explain the presence of a favorable terrain for syncopes and orthostatic hypotension [2] [4] [5] [6] [29]: cardiovascular comorbidities with structural damage to the vascular tree: HBP, atherosclerosis, heart failure, coronary disease, hypotensive drugs, diabetes mellitus, carotid sinus disease, failure in baroreflexes with autonomic insufficiency, Parkinson's disease, postprandial hypotension and autonomic peripheral neuropathy. All of them favor the occurrence of syncope and $\mathrm{OH}$ in older adults [2] [5] [6] [29]. Even if HBP [30] [31] [32] coexists. 
The most frequent combinations in the same patient were: HBP plus hypotensive drugs (n: 13), HBP plus diabetes mellitus plus hypotensive drugs (n: 4), diabetes mellitus plus hypotensive drugs (n: 2), diabetes mellitus plus failure in baroreflexes (n: 6), HBP plus diabetes mellitus (7) and finally HBP plus failure in the baroreflexes (n: 4).

\section{Hypotensive Drugs}

Patients report that, when starting using a medication, fainting started or worsened, and stopped when they stopped using it. We can confirm it, by testing withdrawal or reducing doses. This occurred in 8 of 22 cases (36\%) [8] [33] [34] [35] [36].

The effect of the sum of antihypertensive drugs in combination with psychotropic, anxiolytic, antidepressant drugs and the production of $\mathrm{OH}$ and syncope is clear [37].

Orthostatic hypotension is caused by failure of the mechanisms that compensate for the drop in venous return produced when standing. When $\mathrm{OH}$ is recent you should look for polypharmacy, or dehydration.

When it is chronic, it is more likely to find failure in the baroreflexes (worse after 65 years) [38] [39] [40], heart failure, arterial thickening, cardiovascular diseases, and central or peripheral neurological disorders that damage the autonomic nervous system such as diabetic neuropathy autonomic, and/or peripheral, idiopathic neuropathy [41] [42] [43] [44] or Parkinson's disease [44] [45]. Even worse if you add hypotensive drugs [46].

The factors found, concomitant with the $\mathrm{OH}$ [41] [47] [48] [49] are in Table 6.

$\mathrm{OH}$, also asymptomatic, brings an increase in mortality and major cardiovascular events [50] [51] [52] [53].

We found 2 patients with joint ligamentous hypermobility $(\geq 7)$, despite exceeding 50 years old [54] [55] [56]. In one of them, this was the only associated finding. The other patients also suffered from failure of the baroreflexes.

Situational syncope two cases. One of them presented a "cough syncope". The other case was a "gelastic syncope". Both are rare causes of VVS syncope, produced only in situations associated with the Valsalva's manouver [57] [58].

Postprandial hypotension (9.6\% of our cases) is common in geriatric patients and is a little-known cause of syncope [59] [60]. Patients with neurological diseases, autonomic failure, diabetics, hypertensive or dialysis patients are at risk [59] [60]. It is due to an inadequate sympathetic response to splanchnic vasodilation produced during feeding.

Syncope due to compression of the carotid sinus, is usually mentioned as a cause of syncope in the elderly [11] [61] [62], but at least in our sample, was rare.

In 6 patients (11\%), despite follow-up, their syncope was unexplained.

Three of them consulted for a single syncope and the others for a maximum of 2 or 3 episodes. We believe that in these patients, circumstances like ambient 
heat, dehydration or prolonged standing came together at a certain moment: But during follow-up, they have not been repeated [63] [64] [65].

In this subgroup, it is mandatory to determine if they have a heart condition, since they have up to $30 \%$ more mortality than the population of the same age [2] [26]. So, our protocol includes carrying out a complete cardiological evaluation previous to HUT.

\section{Study Limitations}

First, it is difficult to obtain statistically valid conclusions due to the small number of patients studied in our sample. We understand that a greater sample is necessary in the future.

Second, we depend on the good memory of the patient or his relatives to recall that they are consulting for the first syncopal episode. In some cases, the patient, after a second interrogation, remembers having suffered episodes during childhood or adolescence (66).

Third, altough an exhaustive interrogation and pre-HUT exams, it is not always possible to completely rule out other causes such as drowsiness, vertigo, orthostatic dizziness or accidental falls.

\section{In Sum}

It is not common to begin with syncopes over age of 50 .

Finding the ultimate cause for syncope in these patients can be difficult given the multiplicity of interacting factors. They are mainly male and exhibit different causes than those found at a young age.

Its study is more exhaustive and requires a good anamnesis, knowing the drugs used by the patient, knowing the concomitant diseases and careful monitoring to get closer to the diagnosis. We observe the high frequency of orthostatic hypotension as a cause for syncope, which is attributable in many cases to advanced age, failure of baroreflexes, cardiovascular and neurological diseases, and polypharmacy [29] [66] [67].

\section{Acknowledgements}

Cardiology technician personnel: Erika Collío, Pilar Altamirano, Francisco Osorio and Liliana Huerta. Cardiology nurses: Leonor Acevedo and Clotilde Sanhueza. Cardiology Counseling Dr. José Pardo Gutiérrez.

\section{Conflicts of Interest}

The authors declare no conflicts of interest regarding the publication of this paper.

\section{References}

[1] Sheldon, R.S., Sheldon, A.G., Connolly, S.J., Morrillo, C.A., Klingenheben, T., Krahn, A.D., Koshman, M.-L. and Ritchie, D. (2006) Age of First Faint in Patients 
with Vasovagal Syncope. Journal of Cardiovascular Electrophysiology, 17, 49-54. https://doi.org/10.1111/j.1540-8167.2005.00267.x

[2] Savage, D.D., Corwin, L., Mcgee, D.L., Kannel, W.B. and Wolf, P.A. (1985) Epidemiologic Features of Isolated Syncope: The Framingham Study. Stroke, 16, 626-629. https://www.ncbi.nlm.nih.gov/pubmed/4024175 https://doi.org/10.1161/01.STR.16.4.626

[3] Moya, A., Sutton, R., Ammirati, F., Blanc, J.J., Brignole, M., Dahm, J.B., Deharo, J.C., et al. (2009) Guidelines for the Diagnosis and Management of Syncope. European Heart Journal, 30, 2631-2671.

[4] Colman, N., Nahm, K., Ganzeboom, K.S., Shen, W.K., Reitsma, J. Linzer, B., Wieling, W. and Kaufmann, H. (2004) Epidemiology of Reflex Syncope. Clinical Autonomic Research, 14, i9-i17. https://doi.org/10.1007/s10286-004-1003-3

[5] Rose, M.F. and Da Silva, L. (2014) Syncope: Epidemiology, Etiology, and Prognosis. Frontiers in Physiology, 5, 471.

https://www.ncbi.nlm.nih.gov/pmc/articles/pmc4258989/

[6] Soteriades, E.S., Evans, J.C., Larson, M.G., Chen, M.H., Chen, L., Benjamin, E.J. and Levy, D. (2002) Incidence and Prognosis of Syncope. The New England Journal of Medicine, 347, 878-885. https://doi.org/10.1007/s10286-004-1003-3

[7] Zagaria, M.A.E. (2012) Syncope: Medications as Cause and Contributing Factors. US Pharmacist, 37, 22-27.

https://www.uspharmacist.com/article/syncope-medications-as-cause-and-contribu ting-factors

[8] Kenny, R.A. (2010) Syncope. In: Fillit, H.M., Rockwood, K. and Woodhouse, K., Eds., Brocklehurst's Textbook of Geriatric Medicine and Gerontology, 7th Edition, Saunders, Philadelphia, 338-347.

[9] Pirozzi, G., Ferro, G., Langellotto, A., Della-Morte, D., Galizia, G., Gargiulo, G., Cacciatore, F., Ungar, A. and Abete, P. (2013) Syncope in the Elderly: An Update. Journal of Clinical Gerontology \& Geriatrics, 4, 69-74. https://www.sciencedirect.com/science/article/pii/s2210833513000592 https://doi.org/10.1016/j.jcgg.2013.07.001

[10] Del Rosso, A., Bartoli, P., Bartoletti, A., Brandinelli-Geri, A., Bonechi, F., Maioli, M., et al. (1998) Shortened Head-Up Tilt Testing Potentiated with Sublingual Nitroglycerin in Patients with Unexplained Syncope. American Heart Journal, 135, 564-570. https://doi.org/10.1016/S0002-8703(98)70268-6

[11] Masson, C. (2006) Carotid Sinus Hypersensitivity: An Age-Related Phenomenon. Journal of Neurology, Neurosurgery \& Psychiatry, 77, 1207. https://doi.org/10.1136/jnnp.2006.098442

[12] Rovasi, G., Rafanelli, M. and Ungar, A. (2018) Usefulness of Tilt Testing and Carotid Sinus Massage for Evaluating Reflex Syncope. American Journal of Cardiology, 122, 517-520. https://doi.org/10.1016/j.amjcard.2018.04.033

[13] Seifer, C. (2013) Carotid Sinus Syndrome. Cardiology Clinics, 31, 111-121. https://doi.org/10.1016/j.ccl.2012.10.002

[14] Kenny, R.J., Bhangu, J. and King-Kallimanis, B.L. (2013) Epidemiology of Syncope/Collapse in Younger and Older Western Patient Populations. Progress in Cardiovascular Diseases, 55, 357-363.

https://www.sciencedirect.com/science/article/abs/pii/S0033062012002137?via\%3Di hub

https://doi.org/10.1016/j.ccl.2012.10.002 
[15] Ganzeboom, K.S., Colman, N., Reitsma, J.B., Shen, W.K. and Wieling, W. (2003) Prevalence and Triggers of Syncope in Medical Students. American Journal of Cardiology, 9, 1006-1008. https://doi.org/10.1016/S0002-9149(03)00127-9

[16] Simova, I. (2015) Role of Tilt-Table Testing in Syncope Diagnosis and Management. E-Journal of Cardiology Practice, 13.

https://www.escardio.org/Journals/E-Journal-of-Cardiology-Practice/Volume-13/ro le-of-tilt-table-testing-in-syncope-diagnosis-and-management

[17] Marrison, V.K., Fletcher, A. and Parry, S.W. (2012) The Older Patient with Syncope: Practicalities and Controversies. International Journal of Cardiology, 155, 9-13.

https://www.internationaljournalofcardiology.com/article/S0167-5273(10)00903-4/f $\underline{\text { ulltext }}$

[18] Matthews, I.G., Tresham, I.A.E. and Parry, S.W. (2015) Syncope in the Older Person. Cardiology Clinics, 33, 411-421.

https://www.sciencedirect.com/science/article/abs/pii/S0733865115000363?via\%3Di hub

[19] The Consensus Committee of the American Autonomic Society and the American Academy of Neurology (1996) Consensus Statement on the Definition of Orthostatic Hypotension, Pure Autonomic Failure, and Multiple System Atrophy. Neurology, 46, 1470. https://doi.org/10.1212/WNL.46.5.1470

[20] Gibbons, C.H. and Freeman, R. (2015) Clinical Implications of Delayed Orthostatic Hypotension. A 10-Year Follow-Up Study. Neurology, 85, 1632-1637.

https://n.neurology.org/content/85/16/1362 https://doi.org/10.1212/WNL.0000000000002030

[21] Ruwald, M.H. (2018) Syncope and Orthostatic Hypotension: Early Markers of Cardiac Disease in the General Population. Heart, 104, 456-457. https://doi.org/10.1136/heartjnl-2017-312112

[22] Testa, G., Ceccofiglio, A., Mussi, C., Bellelli, G., Nicosia, F., Bo, M., Riccio, D., Curcio, F., Martone, A.M., Noro, G., Landi, F., Ungar, A., and Abete, P. (2018) Hypotensive Drugs and Syncope Due to Orthostatic Hypotension in Older Adults with Dementia (Syncope and Dementia Study). Journal of the American Geriatrics Society, 66, 1532-1537. https://doi.org/10.1111/jgs.15421

[23] Cheshire Jr., W.P. and Goldstein, D.S. (2019) Autonomic Uprising: The Tilt Table Test in Autonomic Medicine. Clinical Autonomic Research, 29, 215-230. https://doi.org/10.1007/s10286-019-00598-9

[24] Pstras, L., Thomaseth, K., Waniewski, J., Balzani, I. and Bellavere, F. (2015) The Valsalva Manouvre: Physiology and Clinical Examples. Acta Physiologica, 217, 103-119. https://doi.org/10.1111/apha.12639

[25] Wada, N., Singer, W., Gehrking, T.L., Sletten, D.M. and Schmelzer, J.D. and Low P.A. (2014) Comparison of Baroreflex Sensitivity with a Fall and Rise in Blood Pressure Induced by the Valsalva Manouvre. Clinical Science, 127, 307-313. https://doi.org/10.1042/CS20130802

[26] Brignole, M. (2007) Diagnosis and Treatment of Syncope. Heart, 93, 130-136. https://www.ncbi.nlm.nih.gov/pmc/articles/PMC1861366/ https://doi.org/10.1136/hrt.2005.080713

[27] Lo, R. and Cohen, T.J. (2007) Laughter-Induced Syncope: No Laughing Matter. The American Journal of Medicine, 120, E5. https://www.amjmed.com/article/s0002-9343(06)00898-9/fulltext. https://doi.org/10.1016/j.amjmed.2006.07.019 
[28] Hilz, M.J., Marthol, H. and Neundörfer, B. (2002) Syncope-A Systematic Overview of Classification Pathogenesis Diagnosis and Management. Fortschritte der Neurologie-Psychiatrie, 70, 95-107. https://doi.org/10.1055/s-2002-19923

[29] Edwards, F.J. and Winograd, S.M. (2018) Syncope. https://www.reliasmedia.com/articles/143323-syncope

[30] Patel, P.R. and Quinn, J.V. (2015) Syncope: A Review of Emergency Department Management and Disposition. Clinical and Experimental Emergency Medicine, 2, 67-74. https://www.ncbi.nlm.nih.gov/pmc/articles/PMC5052859/ https://doi.org/10.15441/ceem.14.049

[31] Mukai, S. and Lipsitz, L.A. (2002) Orthostatic Hypotension. Falls and Syncope in Elderly Patients. Clinics in Geriatric Medicine, 18, 253-268. https://doi.org/10.1016/S0749-0690(02)00008-3

[32] Youde, J., Ruse, C., Parker, S. and Fotherby, M. (2000) A High Diagnostic Rate in Older Patients Attending an Integrated Syncope Clinic. Journal of the American Geriatrics Society, 48, 783-787. https://doi.org/10.1111/j.1532-5415.2000.tb04753.x

[33] O’Brien, H. and Kenny, A.R. (2014) Syncope in the Elderly. European Cardiology Review, 9, 28-36. https://www.ncbi.nlm.nih.gov/pmc/articles/PMC6159456/ https://doi.org/10.15420/ecr.2014.9.1.28

[34] Tykocki, T., Guzek, K. and Nauman, P. (2010) Orthostatic Hypotension and Supine Hypertension in Primary Autonomic Failure. Pathophysiology, Diagnosis, and Treatment. Kardiologia Polska, 68, 1057-1063.

[35] Goldstein, D.S., Pechnik, S., Holmes, C., Eldadah, B. and Sharabi, Y. (2003) Association between Supine Hypertension and Orthostatic Hypotension in Autonomic Failure. Hypertension, 42, 136-142. https://doi.org/10.1161/01.HYP.0000081216.11623.C3

[36] Biaggioni, I., Shibao, C.A., Diedrich, A., Muldowney, J.A.S., Laffer, C.L. and Jordan, J. (2019) Blood Pressure Management in Afferent Baroreflex Failure: JACC Review Topic of the Week. Journal of the American College of Cardiology, 74, 2939-2947. https://doi.org/10.1016/j.jacc.2019.10.027

[37] NINDS, Syncope Information Page. National Institute of Neurological Disorders and Stroke. National Institutes of Health. Updated October 4, 2011. https://www.ninds.nih.gov/Disorders/All-Disorders/Syncope-Information-Page

[38] Morag, R. (2017) Syncope. Medscape. https://emedicine.medscape.com/article/811669-overview

[39] Saseen, J.J. and Mclaughlin, E.J. (2008) Hypertension. In: Dipiro, J.T., Talbert, R.L., Yee, G.C., et al., Eds., Pharmacotherapy: A Pathophysiologic Approach, 7th Edition, McGraw-Hill Inc., New York, 56.

[40] Crimson, M.L., Argo, T.R. and Buckey, P.F. (2008) Schizophrenia. In: Dipiro, J.T., Talbert, R.L., Yee, G.C., et al., Eds., Pharmacotherapy: A Pathophysiologic Approach, 7th Edition. McGraw-Hill Inc., New York, 1111.

[41] Ruwald, M.H., Hansen, M.L., Lamberts, M., et al. (2013) Comparison of Incidence, Predictors, and the Impact of Co-Morbidity and Polypharmacy on the Risk of Recurrent Syncope in Patients $<85$ versus $\geq 85$ Years of Age. American Journal of Cardiology, 112, 1610-1615. https://doi.org/10.1016/j.amjcard.2013.07.041

[42] Fedorowski, A. and Melander, O. (2013) Syndromes of Orthostatic Intolerance: A Hidden Danger. Journal of Internal Medicine, 273, 322-335. https://doi.org/10.1111/joim.12021

[43] Chaddha, A., Wenzke, K.E., Brignole, M., Wasmund, S.L., Page, R.L. and Hamdan, 
M.H. (2016) The Role of the Baroreflex in Tilt Table Testing: Outcome and Type of Response. JACC: Clinical Electrophysiology, 2, 812-817. https://doi.org/10.1016/j.jacep.2016.05.001

[44] Abuzinadah, A.R., Sinn, D.I., Freeman, R. and Gibbons, C.H. (2016) Blood Pressure Oscillations in Baroreflex Failure. Clinical Autonomic Research, 26, 465-466. https://doi.org/10.1007/s10286-016-0374-6

[45] Baker, S.E., Limberg, J.K., Dillon, G., Curry, T.B. and Joyner, M.J. (2018) Aging Alters the Relative Contributions of the Sympathetic and Parasympathetic Nervous System to Blood Pressure Control in Women. Hypertension, 72, 1236-1242. https://doi.org/10.1161/HYPERTENSIONAHA.118.11550

[46] Credeur, D.P., Holwerda, S.W., Boyle, L.J., Vianna, L.C., Jensen, A.K. and Fadel, P.J. (2014) Effect of Aging on Carotid Baroreflex Control of Blood Pressure and Leg Vascular Conductance in Women. American Journal of Physiology-Heart and Circulatory Physiology, 306, H1417-1425. https://pubmed.ncbi.nlm.nih.gov/24682393/ https://doi.org/10.1152/ajpheart.00036.2014

[47] Teixeira, S.C., Madureira, J.B., Azevedo, E.I. and Castro, P.M. (2019) Ageing Effects the Balance between Central and Peripheral Mechanisms of Cerebrovascular Regulation with Increasing Influence of Systolic Blood Pressure Levels. European Journal of Applied Physiology, 119, 519-529. https://doi.org/10.1007/s00421-018-4036-3

[48] Gupta, V. and Lipsitz, L.A. (2007) Orthostatic Hypotension in the Elderly: Diagnosis and Treatment. The American Journal of Medicine, 120, 841-847.

https://doi.org/10.1016/j.amjmed.2007.02.023

[49] Strano, S., Fanciulli, A., Rizzo, M., Marinelli, P., Palange, P., Tiple, D., De Vincentis, G., Calcagnini, G., Censi, F., Meco, G. and Colosimo, C. (2016) Cardiovascular Dysfunction in Untreated Parkinson's Disease: A Multi-Modality Assessment. Journal of the Neurological Sciences, 370, 251-255. https://doi.org/10.1016/j.jns.2016.09.036

[50] Ruwald, M.H., Ricci, F., Fedorowski, A. and Radico, F. (2015) Cardiovascular Morbidity and Mortality Related to Orthostatic Hypotension: A Meta-Analysis of Prospective Observational Studies. European Heart Journal, 36, 1609-1617. https://doi.org/10.1093/eurheartj/ehv093

[51] Fedorowski, A., Stavenow, L. and Hedblad, B. (2010) Orthostatic Hypotension Predicts All-Cause Mortality and Coronary Events in Middle-Aged Individuals (The Malmö Preventive Project). European Heart Journal, 31, 85-91. https://doi.org/10.1093/eurheartj/ehp329

[52] Kaufmann, H., Norcliffe-Kaufmann, L. and Palma, J.A. (2020) Baroreflex Dysfunction. The New England Journal of Medicine, 382, 163-178. https://doi.org/10.1056/NEJMra1509723

[53] Lipsitz, L.A. (1989) Orthostatic Hypotension in the Elderly. The New England Journal of Medicine, 321, 952-957. https://www.nejm.org/doi/full/10.1056/NEJM198910053211407?url_ver=Z39.88-20 03\&rfr_id=ori:rid:crossref.org\&rfr_dat=cr_pub\%3dpubmed https://doi.org/10.1056/NEJM198910053211407

[54] Mader, S.L. (1989) Aging and Postural Hypotension. An Update. Journal of the American Geriatrics Society, 37, 129-137. https://onlinelibrary.wiley.com/doi/pdf/10.1111/j.1532-5415.1989.tb05871.x https://doi.org/10.1111/j.1532-5415.1989.tb05871.x

[55] Mathias, C.J. (1995) Orthostatic Hypotension: Causes, Mechanisms, and Influencing Factors. Neurology, 45, S6-11. https://europepmc.org/article/med/7746371 
[56] Yasa, E., Ricci, F. and Magnusson, M., et al. (2018) Cardiovascular Risk after Hospitalization for Unexplained Syncope and Orthostatic Hypotension. Heart, 104, 487-493. https://doi.org/10.1136/heartjnl-2017-311857

[57] Solbiati, M., Casazza, G., Dipaola, F., et al. (2015) Syncope Recurrence and Mortality: A Systematic Review. EP Europace, 17, 300-308. https://doi.org/10.1093/europace/euu327

[58] Bravo, J.F. (2009) Ehlers-Danlos Syndrome, with Special Emphasis in the Joint Hypermobility Syndrome. Rev Med Chile, 137, 1488-1497.

[59] Gazit, Y., Nahir, A.M, Grahame, R. and Jacob, G. (2003) Dysautonomia in the Joint Hypermobility Syndrome. The American Journal of Medicine, 115, 33-40. https://doi.org/10.1016/S0002-9343(03)00235-3

[60] Celletti, C., Camerota, F., Castori, M., Censi, F., Gioffrè, L., Calcagnini, G. and Strano, S. (2017) Orthostatic Intolerance and Postural Orthostatic Tachycardia Syndrome in Joint Hypermobility Syndrome/Ehlers-Danlos Syndrome, Hypermobility Type: Neurovegetative Dysregulation or Autonomic Failure? BioMed Research International, 2017, Article ID: 9161865. https://doi.org/10.1155/2017/9161865

[61] Luciano, G.L., Brennan, M.J. and Rothberg, M.B. (2010) Postprandial Hypotension. The American Journal of Medicine, 23, 281.E1-281.E6. https://www.amjmed.com/article/S0002-9343(09)00719-0/fulltext https://doi.org/10.1016/j.amjmed.2009.06.026

[62] Vloet, L.C., Pel-Little, R.E., Jansen, P.A., et al. (2005) High Prevalence of Postprandial and Orthostatic Hypotension among Geriatric Patients Admitted to Dutch Hospitals. The Journals of Gerontology: Series A, 60, 1271-1277.

https://www.medscape.com/viewarticle/559578_5 https://doi.org/10.1093/gerona/60.10.1271

[63] Klein, K.M. and Berkovic, S.F. (2014) Genetics of Vasovagal Syncope. Autonomic Neuroscience-Basic \& Clinical, 184, 60-65. https://doi.org/10.1016/j.autneu.2014.03.008

[64] Holmegard, H.N., Benn, M., Kaijer, M., Haunsø, S. and Mehlsen, J. (2013) Prevalence of Family History in Patients with Reflex Syncope. Journal of Clinical Neuroscience, 20, 692-696. https://doi.org/10.1016/j.jocn.2012.03.054

[65] Negrusz-Kawecka, M., BaŃkowski, T., Tabin, M., Paprocka, M., Mercik, A., Misztal, J., Nowak, P., Zysko, D. and Gajek, J. (2012) Familial Predisposition to Vasovagal Syncope. Acta Cardiologica, 67, 279-284. https://doi.org/10.1080/AC.67.3.2160715

[66] Jiménez-Cohl, P., Ortega, G.M., Labrín, E.D., Bruna, V.M., Zumaran, I.R. and Núñez, M.M. (2018) Síncope Único O Muy Poco Frecuente en 104 Casos [Single or Very Isolated Syncope in 104 Cases]. Revista Chilena de Neuro-Psiquiatría, 56, 251-259. https://doi.org/10.4067/s0717-92272018000400251

[67] Chaddha, A., Rafanelli, M., Brignole, M., Sutton, R., Wenzke, K.E., Wasmund, S.L., Page, R.L. and Hamdan, M.H. (2016) The Pathophysiologic Mechanisms Associated with Hypotensive Susceptibility. Clinical Autonomic Research, 26, 261-268. https://doi.org/10.1007/s10286-016-0362-x 